FROM THE EDITOR'S DESK

\title{
From the Editors Desk: Introducing the JGIM Bottom Line Summary and Podcast
}

\author{
Michael E. Bowen, MD, MPH, MSCS ${ }^{7}$, William Fuller, MD², and \\ Devan Kansagara, $M D, M C R^{3,4}$
}

\begin{abstract}
'Department of Internal Medicine, Pediatrics, and Clinical Sciences, Divisions of General Internal Medicine and Outcomes and Health Services Research, University of Texas Southwestern Medical Center, Dallas, TX, USA; ${ }^{2}$ Columbia University Medical Center, New York, USA; ${ }^{3}$ Department of Medicine, Oregon Health and Science University, Portland, USA; ${ }^{4}$ Portland Evidence Synthesis Program, VA Portland Health Care System, Portland, USA.
\end{abstract}

J Gen Intern Med 34(9):1671-2

DOI: $10.1007 / \mathrm{s} 11606-019-05015-5$

(c) Society of General Internal Medicine 2019

$\mathrm{M}$ r. T is a 55-year-old male with left knee osteoarthritis and gradually worsening left knee pain that is not responsive to acetaminophen. He has a close friend Mr. B who takes opioid medications for his knee pain and says that they really help him make it through the day with less pain. Mr. T wonders if he would benefit from opioids as well. You know there is a recent trial that examined this question nested snugly in the 20-deep pile of journals serving as your home office doorstop. You recall a recent Times headline "nonopioid drugs work as well as opioids for arthritis pain," but you do not know the details and feel embarrassed to describe only the headline to your patient. ${ }^{1}$

As general internists, we are committed to the practice of evidence-based medicine which is "about integrating individual clinical expertise and the best external evidence." ${ }^{2}$ This integration is challenging. We must stay abreast of new evidence, distill key findings in order to translate the evidence into practice, and discuss it in language accessible to our patients on the fly during a 10-15-min primary care visit.

In this issue of JGIM, we are excited to introduce a new feature series designed to assist clinicians in this daunting task-the Bottom Line Summary. Bottom Line Summaries are succinct reviews of topical, practice-changing studies that help clinicians integrate evidence into clinical practice. The SGIM Evidencebased Medicine Subcommittee of the Clinical Practice Committee, formerly the Evidence-based Medicine Task Force, has been producing the Bottom Line Summary since 2012. We are excited to transition this series from JGIM Online-only content to JGIM print material to expand its readership and impact.

This series will highlight timely articles that are highly relevant to general internists and hospitalists. Articles can be individual trials or observational studies that represent a major contribution to knowledge in a given field, or systematic reviews of important topics. Selected summaries will be accompanied by podcasts

Published online July 24, 2019 featuring in-depth conversations with content experts discussing study findings in context of related literature.

Key features of the Bottom Line Summary are designed to facilitate visit-based, evidence-driven conversations with patients including:

- Study Description and Synthesis: Succinct, clear, lay language distillation of key study design features and findings to help clinicians quickly grasp and translate important facts about the study.

- Infographic: Key study findings are visually depicted in displays such as bar graphs and icon arrays that accurately represent clinical findings and facilitate understanding in clinical conversations.

- Tips for Discussion with Patients: Study findings of greatest importance to patients are highlighted in succinct, patient-friendly bullet points that are designed to be used as talking points in clinical discussions.

- Methodologic Issues and Application to Practice: Brief descriptions of important methodologic issues using published criteria as a guide and a description of populations and settings to which the evidence does and does not apply.

The Bottom Line Summary series begins in this issue of JGIM by summarizing the Strategies for Prescribing Analgesics Comparative Effectiveness (SPACE) trial which compares the effectiveness of opioid vs. non-opioid medications for the treatment of chronic musculoskeletal pain in opioid-naive patients. ${ }^{3}$ This Bottom Line Summary is also accompanied by the inaugural SGIM Bottom Line Podcast which digs deeper into the nuances of utilizing opiates for chronic musculoskeletal pain. In the coming months, look for additional Bottom Line Summaries tackling the efficacy of steroid injections in the management of osteoarthritis and the role of Coronary Artery Calcium (CAC) testing in risk stratification of patients with elevated cardiovascular risk.

We are excited to launch the Bottom Line Summary as the latest series in JGIM and we look forward to receiving your submissions. Submission guidelines can be found in the JGIM instructions for authors at www.jgim.org. Given the unique nature of this series, interested authors should contact the JGIM Bottom Line Summary Editor to discuss their proposal prior to submission. All submissions will be peer reviewed and published 
in JGIM if accepted. Select summaries will be accompanied by a Bottom Line Summary Podcast to broaden impact and uptake of the summary: contact Bill Fuller at wsfuller@gmail.com, editor of the Bottom Line Summary podcast with questions about or ideas for the podcast. Interested Bottom Line Summary authors should contact Michael Bowen, JGIM Bottom Line Summary Editor at michael.bowen@utsouthwestern.edu.

Corresponding Author: Michael E. Bowen, MD, MPH, MSCS; Department of Internal Medicine, Pediatrics, and Clinical Sciences, Divisions of General Internal Medicine and Outcomes and Health Services ResearchUniversity of Texas Southwestern Medical Center, Dallas, TX, USA (e-mail: michael.bowen@utsouthwestern.edu).

\section{Compliance with Ethical Standards:}

Conflict of Interest: The author declares that he/she does not have a conflict of interest.

\section{REFERENCES}

1. Bakalar N. For arthritis pain, nonopioid drugs work as well as opioids. The New York Times; 2018. Retrieved from https://www.nytimes.com/2018/ 03/07/well/live/for-arthritis-pain-tylenol-works-as-well-as-opioids.html. Accessed 9 Jan 2019.

2. Sackett DL. Evidence based medicine: what it is and what it isn't. BMJ. 1996;312: 71-72.

3. Krebs EE, Gravely A, Nugent $\mathbf{S}$ et al. Effect of opioid vs. nonopioid medications on pain-related function in patients with chronic back pain or hip or knee arthritis pain: the SPACE randomized control trial. JAMA. 2018;319(9):872-882.

Publisher's Note Springer Nature remains neutral with regard to jurisdictional claims in published maps and institutional affiliations. 